\title{
Is Deconfined Quantum Criticality in Frustrated Antiferromagnets Ruled Out by Generic Fluctuation Induced First Order Behavior?
}

\author{
F. Krüger
}

Received: 3 July 2007 / Accepted: 3 July 2007 / Published online: 14 September 2007

(C) Springer Science+Business Media, LLC 2007

\begin{abstract}
Recently, it was argued that quantum phase transitions in frustrated two dimensional antiferromagnets can be radically different from their classical counterparts with as a highlight, the "deconfined critical points" exhibiting fractionalization of quantum numbers due to Berry phase effects. However, field theoretical descriptions rest on a naïve coarse graining of the microscopic lattice model assuming order parameter fluctuations on small scales to be smooth. We have developed a novel renormalization approach for such systems which incorporates fluctuations on small scales in a natural way, and is fully respecting the underlying lattice structure and the frustration mechanism. According to our findings, another profound phenomenon is around the corner: a fluctuation induced first order transition.
\end{abstract}

Keywords Quantum criticality · Antiferromagnetism · Fluctuations

\section{Introduction}

Since the discovery in 1986 of high- $T_{c}$ superconductivity in layered cuprates, quantum phase transitions in twodimensional antiferromagnets have been the subject of intense research [1]. While doping the $\mathrm{CuO}_{2}$ layers with charge carriers, long-range magnetic order is destroyed and superconductivity is induced, magnetic frustration which is

This work was supported by Dutch Science Foundation NWO/FOM.

F. Krüger $(\bowtie)$

Instituut-Lorentz, Universiteit Leiden, P.O. Box 9506, 2300

Leiden, The Netherlands

e-mail: krueger@lorentz.leidenuniv.nl present in the parent compounds has been argued to lead to a stabilization of different paramagnetic ground states like RVB spin-liquid [2] or valence-bond solid (VBS) states [3].

Novel scenarios for phase transitions between Néel order and these exotic phases beyond the Landau-GinzburgWilson (LGW) paradigm have been suggested $[4,5]$ joggling fundamental concepts. The Heisenberg model on a square lattice with antiferromagnetic nearest neighbor couplings $J_{1}$, frustrated by ring exchange or antiferromagnetic next-nearest couplings, $J_{2}$ serves as a prototype for studying magnetic quantum-phase transitions. On a field theoretical level, the description of the long-wavelength dynamics of the $d$ dimensional antiferromagnet in the vicinity of a ground state with collinear long-range Néel order is governed by a non-linear $\sigma$ model (NL $\sigma \mathrm{M})$ in $d+1$ dimensions, as first noted by Haldane [6], where the coupling constant controlling the strength of the quantum fluctuations can be tuned by the frustration in the original lattice model.

For sufficiently strong frustration, quantum fluctuations will eventually destroy long-range magnetic order leading to a quantum phase transition into a paramagnetic (PM) phase. In the absence of long-range Néel order, topological Berry phases which occur naturally in the spin-coherent state path integral representation and augment the NL $\sigma \mathrm{M}$ are no longer negligible. Remarkably, the interference of Berry phases of proliferating topological excitations (monopole tunneling events) can give rise to ground-state degeneracies corresponding to a translation-symmetry breaking by dimerization and formation of valence-bond solid (VBS) phases $[6,7]$.

The Néel-VBS transition has been argued to be in a novel quantum criticality class that does not fit in the standard LGW paradigm. Intriguing data on this transition was obtained in simulations of the $S=1 / 2$ quantum $\mathrm{XY}$ model 
frustrated by ring exchange $[8,9]$. The transition was interpreted as a second-order one; this possibility was predicted by the theory of the deconfined critical point and suggested to be generic for a variety of experimentally relevant twodimensional antiferromagnets [5]. However, in a more careful finite-size analysis of the XY case, it was demonstrated that the Néel-VBS point represents an anomalously weak first-order transition [10]. Frustrated Heisenberg antiferromagnets which cannot be studied by quantum Monte Carlo simulations due to the infamous "sign problem," have been considered in exact diagonalization studies [11], however, because of the limitations to very small lattices, it has not been possible to study phase transitions in detail.

Whereas numerical simulations or exact diagonalization studies are not capable of giving us conclusive answers on the nature of quantum phase transitions in frustrated Heisenberg antiferromagnets, effective field theories capturing the long-wavelength dynamics like the NL $\sigma \mathrm{M}$ suffer from an intrinsic shortcoming: they rest on a naïve coarse graining assuming fluctuations of the Néel order parameter to be smooth on small scales and incorporate the frustration mechanism only in a very crude way. In essence, all kinds of frustrations act on the level of the continuum field theory in the same way: they serve as a knob for tuning the coupling constant controlling the strength of the quantum fluctuations.

In a recent paper [12], we have developed a novel renormalization approach that circumvents the naïve coarse graining procedure and fully respects the underlying lattice geometry and the microscopic frustration mechanism. It combines the systematic treatment of all corrections in order $1 / S$ on the level of the conventional first-order spinwave theory (SWT) with the merits of a renormalization approach, which goes beyond any finite order in $1 / S$ by an infinite iteration of differential steps, successively eliminating the spin-wave modes of highest energy. As a result, we obtain an improved description of the phase transitions. In particular, the possibility of a fluctuation induced first-order transition which is not accessible on the level of the NL $\sigma \mathrm{M}$ [13] is included in a natural way.

\section{Spin-Coherent State Representation and Renormalization Approach}

The key idea of our renormalization approach is a novel kind of path integral quantization, which explicitly keeps the fields from both sublattices and makes it possible for us to treat the effects of umklapp on an equal footing with the spin-wave interactions. To demonstrate the workings of the approach, we stick to the simplest frustration mechanism realized in the aforementioned $J_{1}-J_{2}$ Heisenberg model on a square lattice. Fluctuations are treated in a coherent spin state path-integral representation of the model, where a spin state corresponds to a unit vector $\vec{n}$. In the absence of fluctuations, one expects collinear Néel order to be stable for $\alpha=J_{2} / J_{1}<1 / 2$ and spins would assume the states $\left|\vec{n}_{A / B}\right\rangle=\left| \pm \vec{e}_{z}\right\rangle \stackrel{\wedge}{=}|S, \pm S\rangle$ on the two sublattices, A and B. Using the standard Trotter formula, we obtain an action in imaginary time $\tau$ (discretized in intervals of duration $\Delta \tau$ ) [1]. We start from a stereographic parametrization of coherent states and expand the discrete imaginary time action in terms of the complex stereographic fields $a$ and $b$ (see [12] for details).

To leading order in $1 / S$, the fluctuations are controlled by the bilinear part $S_{0}$ of the action that represents free magnons. We also retain the quartic contribution $S_{\text {int }}$ to the action, which represents magnon interactions and contain the renormalization of single-magnon parameters of relative order $1 / S$. The single-magnon contribution in terms of the stereographic fields $a, b$ and their complex conjugated $\bar{a}, \bar{b}$ is given in momentum space by

$$
\begin{aligned}
S_{0}= & \sum_{\tau} \int_{k}\left\{\frac{1}{2 g}\left[\bar{a}_{k} \Delta a_{k}-\Delta \bar{a}_{k} a_{k}^{\prime}+\bar{b}_{k} \Delta b_{k}-\Delta \bar{b}_{k} b_{k}^{\prime}\right]\right. \\
& \left.+\Delta \tau S\left[\Gamma_{k}^{+}\left(\bar{a}_{k} a_{k}^{\prime}+\bar{b}_{k} b_{k}^{\prime}\right)+\Gamma_{k}^{-}\left(\bar{a}_{k} \bar{b}_{k}+a_{k}^{\prime} b_{k}^{\prime}\right)\right]\right\},
\end{aligned}
$$

with exchange couplings $\Gamma_{k}^{+}=J_{k}^{+}-J_{0}^{+}+J_{0}^{-}, \Gamma_{k}^{-}=$ $J_{k}^{-}=2 J_{1}\left[\cos \left(k_{x}\right)+\cos \left(k_{y}\right)\right]$ and $J_{k}^{+}=2 J_{2}\left[\cos \left(k_{x}+k_{y}\right)+\right.$ $\left.\cos \left(k_{x}-k_{y}\right)\right]$. Here, the primes denote a time shift $\tau \rightarrow$ $\tau-\Delta \tau$ and $\Delta \phi_{k}=\phi_{k}-\phi_{k}^{\prime}$ for $\phi=a, b$. The dimensionless parameter $g$ represents the strength of quantum fluctuations. It assumes the value $g=1$ in the unrenormalized model and turns out to increase under renormalization.

Diagonalizing this bilinear action, one easily obtains the magnon dispersion $\varepsilon_{k}=S g \sqrt{\left(\Gamma_{k}^{+}\right)^{2}-\left(\Gamma_{k}^{-}\right)^{2}}$. For $\alpha<1 / 2$, the low-energy spin-wave excitations are characterized by an isotropic dispersion $\varepsilon_{k}=c|k|+O\left(k^{2}\right)$ with a spin-wave velocity $c=2 \sqrt{2} g S J_{1} \sqrt{1-2 \alpha}$. Likewise, the exchange couplings generate spin stiffness $\rho=S^{2} J_{1}(1-2 \alpha)$ for lowenergy modes. For strong frustration $(\alpha>1 / 2)$, the stiffness becomes negative and the spin-wave velocity is ill defined due to the presence of unstable modes in the center of the BZ (see Fig. 1). From the diagonalization procedure, we also obtain the correlators of fields from the same sublattice or from different sublattices taken at the same time or shifted by one time step [12].

Starting from this action with bilinear and quartic terms, we implement a renormalization procedure as follows. In successive steps, the modes of highest energy (an infinitesimal fraction of all modes) are integrated out. This decimation of modes yields an effective theory for the remaining modes and gives rise to differential flow equations [12]. As flow parameter, we choose $l=\frac{1}{2} \ln \left(A_{B Z} / A_{R B Z}\right)$, where 
Fig. 1 (Colour online)

Evolution of modes under coarse graining. Each panel corresponds to the area

$\left|k_{x}\right| \leq \pi,\left|k_{y}\right| \leq \pi$. Red color represents small, yellow high positive energy. Black lines are lines of constant energy, blue areas represent unstable modes. Panels a-d: in the Néel phase the RBZ may become disconnected first, then always shrinks to a sphere (here $S=1 / 2, \alpha=0.3$,

$l=0,0.25,0.5,1.0)$. Panels e-h: in the Néel phase for strong frustration $\alpha>1 / 2$, initially unstable modes (blue area) are renormalized to stable modes and the RBZ eventually also shrinks to a sphere (here $S=1 / 2, \alpha=0.55$, $l=0,0.11,0.29,1.30$ ). Panels i-k: in the columnar phase, after the elimination of all stable modes, an area of unstable modes survives (here $S=2$, $\alpha=0.6, l=0,0.10,0.20$ )
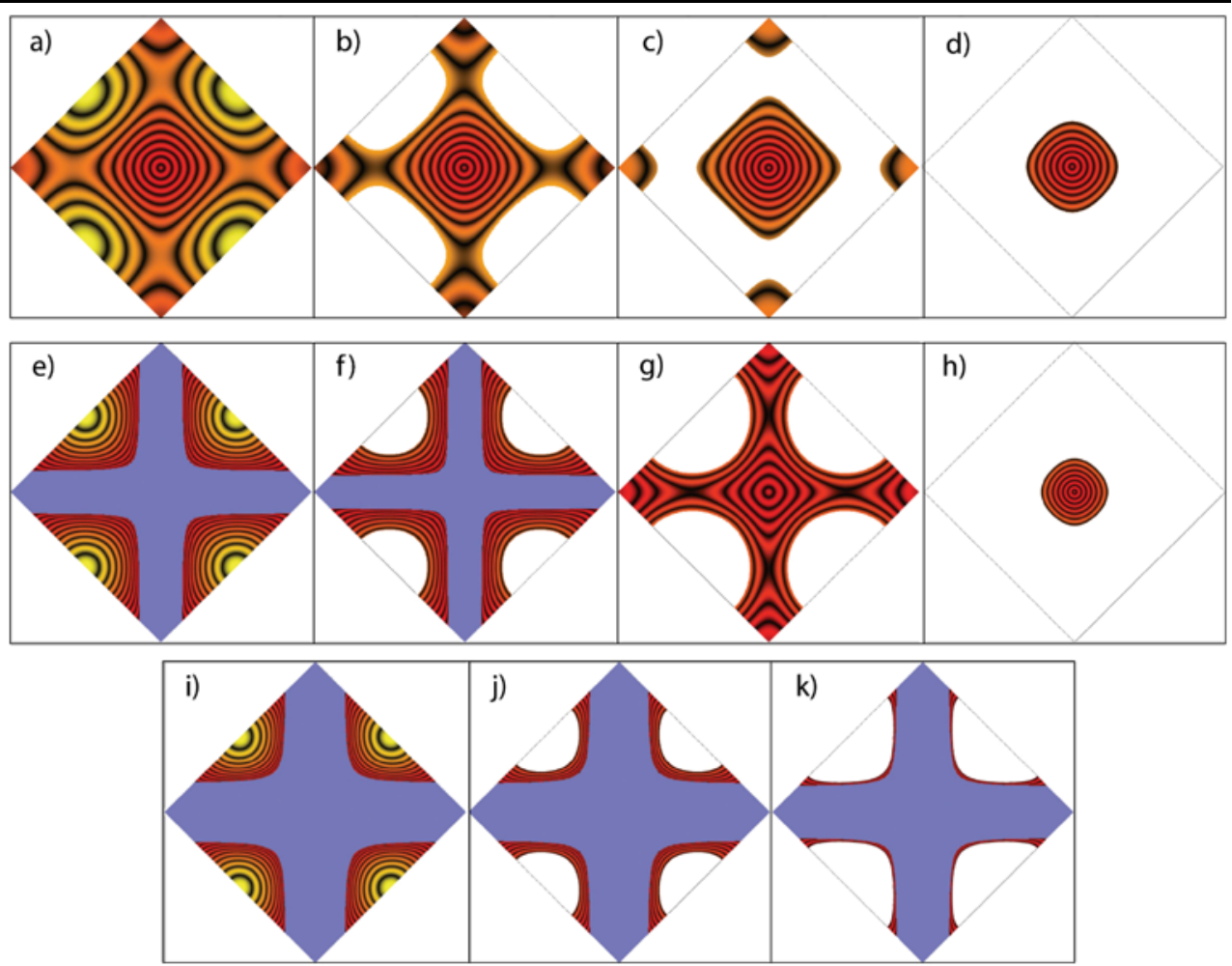

$A_{B Z}=2 \pi^{2}$ is the area of the original $\mathrm{BZ}$, and $A_{R B Z}$ is the area of the residual BZ (RBZ) populated by the remaining modes. The evolution of the RBZ and the singlemagnon-dispersion is illustrated in Fig. 1 for various parameters.

The nature of magnetic order can be identified from the flow behavior. Three possibilities are observed. (i) The RBZ shrinks to a circle of decreasing radius $\propto e^{-l}$ [see Fig. 1, panels $(\mathrm{a}-\mathrm{d})$ and panels $(\mathrm{e}-\mathrm{h})]$ while $J_{1,2}$ and $g$ (as well as the derived quantities $c, \rho, \alpha)$ converge to positive values. In this asymptotic large scale limit, our flow equations match with those derived from the NL $\sigma \mathrm{M}$ [13]. Then, Néel order is present, characterized by these renormalized lowenergy parameters. Remarkably, for sufficiently large $S$, we find Néel order to be stable above the classical threshold $\alpha=1 / 2$. In this region, initially unstable long-wavelength modes are renormalized to stable ones by spin-wave interactions on small and intermediate scales [see Fig. 1, panels (e-h)]. (ii) At some finite $l^{*}$, fluctuations become so strong that $g$ diverges and $J_{1,2}$ vanish. This indicates the loss of magnetic order due to overwhelming quantum fluctuations. (iii) For strong frustration, it is also possible that a finite RBZ of unstable modes remains after decimation of all stable modes (see Fig. 1, panels (i-k)). This indicates the instability toward a discontinuous first transition.

The resulting phase diagram is shown in Fig. 2. While the phase boundary is located at academically small spin values for small frustration, $S$ reaches physically meaningful values at larger frustration. We find the Néel phase to reach up

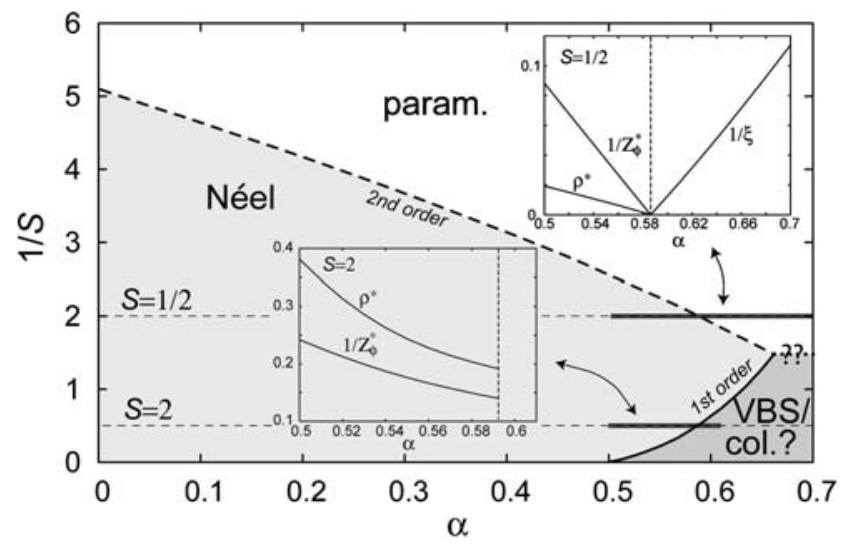

Fig. 2 Phase diagram showing the 2nd order transition line between the Néel ordered and the PM phase (dashed line) and the 1st order boundary between the Néel phase and adjacent phases where different types of ordering are present (solid line). The nature of these phases cannot be obtained within our approach. Insets: evaluation of the renormalized parameters $Z_{\phi}^{*}=g^{*} / \sqrt{1-2 \alpha^{*}}$ and $\rho^{*}$ in the vicinity of the transitions for $S=1 / 2$ and $S=2$, respectively

to $\alpha \approx 0.66$ for $S=0.68$. For spins smaller than this value, the Néel phase becomes unstable toward a PM phase via a second-order transition, whereas it becomes unstable for $S>0.68$ via a first order transition. Since we deal with a discontinuous transition, the type of ordering in the adjacent phase (dark shaded region in Fig. 2) cannot be obtained within our approach. 


\section{Conclusion}

We have presented a novel renormalization approach for frustrated quantum antiferromagnets which fully accounts for the underlying lattice geometry and consistently captures the renormalization of the single-magnon parameters by spin-wave interactions all over the magnetic BZ.

So far, we applied our approach only to the $J_{1}-J_{2}$ model finding generic fluctuation induced first-order behavior for all spin values except for $S=1 / 2$, where our approach falls short because of the large $S$ expansion.

In conclusion, our approach provides a powerful tool to decide for a given microscopic frustration mechanisms whether small-scale fluctuations drive the system toward a first order transition or if the effective long-wavelength theory becomes applicable. In the latter case, subtle quantum interference effects in the vicinity of a continuous transition may then give rise to deconfined quantum criticality $[4,5]$.

\section{References}

1. Sachdev, S.: Quantum Phase Transitions. Cambridge University Press, Cambridge (1999)

2. Anderson, P.W.: Science 235, 1196 (1987)

3. Read, N., Sachdev, S.: Phys. Rev. Lett. 62, 1694 (1989)

4. Senthil, T., Balents, L., Sachdev, S., Vishwanath, A., Fisher, M.P.A.: Phys. Rev. B 70, 144407 (2004)

5. Senthil, T., Vishwanath, A., Balents, L., Sachdev, S., Fisher, M.P.A.: Science 303, 1490 (2004)

6. Haldane, F.D.M.: Phys. Rev. Lett. 61, 1029 (1988)

7. Fradkin, E., Stone, M.: Phys. Rev. B 38, R7215 (1988)

8. Sandvik, A.W., Daul, S., Singh, R.R.P., Scalapino, D.J.: Phys. Rev. Lett. 89, 247201 (2002)

9. Melko, R.G., Sandvik, A.W., Scalapino, D.J.: Phys. Rev. B 38, R9335 (2004)

10. Kuklov, A., Prokof'ev, N., Svistunov, B.: Phys. Rev. Lett. 93, 230402 (2004)

11. Poilblanc, D., Läuchli, A., Mambrini, M., Mila, F.: Phys. Rev. B 73, 100403R (2006)

12. Küger, F., Scheidl, S.: Europhys. Lett. 74, 896 (2006)

13. Chakravarty, S., Halperin, B., Nelson, D.R.: Phys. Rev. B 39, 2344 (1989) 